\title{
Editorial
}

\section{Design, Dimensioning, and Optimization of 4G/5G Wireless Communication Networks}

\author{
Mariusz Gląbowski, ${ }^{1}$ Haris Gacanin, ${ }^{2}$ Ioannis Moscholios, ${ }^{3}$ and Piotr Zwierzykowski ${ }^{1}$ \\ ${ }^{1}$ Poznan University of Technology, Poznan, Poland \\ ${ }^{2}$ Nokia, Antwerp, Belgium \\ ${ }^{3}$ University of Peloponnese, Tripoli, Greece \\ Correspondence should be addressed to Mariusz Głąbowski; mariusz.glabowski@put.poznan.pl
}

Received 22 January 2017; Accepted 23 January 2017; Published 15 March 2017

Copyright ( 2017 Mariusz Głąbowski et al. This is an open access article distributed under the Creative Commons Attribution License, which permits unrestricted use, distribution, and reproduction in any medium, provided the original work is properly cited.

Designing, dimensioning, and optimization of communication networks have been an inseparable part of the development of telecommunications and information infrastructure from the very beginning of their existence. These networking problems have changed substantially over the recent years as a result of the changes in the telecommunications area towards $4 \mathrm{G} / 5 \mathrm{G}$ wireless multiservice networks and mobile communications, as well as networks convergence. Each and every newly introduced type of a radio network technology is followed by a substantial increase in both the number and the complexity of problems that need to be resolved by theoreticians and engineers.

No matter what these developing changes may bring, the essential tasks for $4 \mathrm{G} / 5 \mathrm{G}$ wireless communication networks remain the same: (1) to develop new technologies offering increasing radio network capacity, (2) to determine and evaluate the relationship between the quality of service (quality of experience) parameters and the parameters characterizing traffic sources (services), (3) to control and optimize the usage of radio network resources, and (4) to enhance the capabilities of data transport, transmission, and reception between end users and the core network. These tasks provide a basis for developing engineering algorithms and tools used for designing, analysis, dimensioning, and optimization of wireless systems and networks.

The objective of this special issue, containing 11 papers selected from submissions to the open call for papers, was to bring together the state-of-the-art research contributions that address challenges in 4G/5G radio networks design, dimensioning, and optimization.

In the paper "Energy Efficiency and Capacity Tradeoff in Cloud Radio Access Network of High-Speed Railways," S. $\mathrm{Li}$ et al. propose a predictable path loss based time domain power allocation (PPTPA) method to improve energy efficiency performance of High-Speed Railways (HSR) communication system. The authors assumed in the paper that in the future the communication system for HSR will be based on cloud radio access network. The optimization problem formulated in the paper is based on joint energy efficiency and services transmission delay constraints. The effectiveness of the proposed power allocation algorithm was validated in the paper by HSR channel measurement trace based emulation results and extensive simulation results.

The paper "Mobile Network Planning Tool Based on Data Analytics" by J. Moysen et al. describes the tool which is based on the information available in the network and allow properly deploying, configuring, and optimizing network nodes. In the tool two optimization techniques, that is, Machine Learning (ML) and Genetic Algorithms (GAs), are used. In order to evaluate the proposed tools two case studies were discussed: for the small cell deployment in a dense indoor scenario and for a detected fault in a macrocell network. A simulation-based performance analysis of the proposed tool was carried out over the Ns-3 LTE-EPC Network Simulator.

In the paper "Virtualized ANR to Manage Resources for Optimization of Neighbour Cell Lists in 5G Mobile Wireless 
Networks" by Y. Shin and S. Kim the network function virtualization (NFV) for Automatic Neighbour Relation (ANR) was proposed. The author assumed that in $5 \mathrm{G}$ networks an increase of simultaneous handover and the change of neighbour cell lists will be very quickly and the traditional ANR function has neighbour removal function but it does not consider fast changes in the neighbour list. Therefore the new ANR-virtual network function (ANR-VNF) is proposed in the paper. The experiments for the studied strategy of ANR were executed in practice by using LTE core network (Amarisoft LTE-100 Platform).

In the paper "A Categorized Resource Sharing Mechanism for Device-to-Device Communications in Cellular Networks" by J. Chen et al., the resource sharing mechanism for device-to-device (D2D) communications is studied. In the proposed solution the D2D pairs are divided into three groups based on comparison of the minimum transmit power with the maximum transmit power of each cellular UE. The mechanism studied in the paper enables multiple D2D pairs in the second group to share the resource with cellular user equipment (UE) simultaneously and D2D pairs in the first group and the third group share resource with cellular UEs based on the transmit power minimization principle. Results of simulations conducted by the authors show that the proposed scheme allows obtaining a relatively higher network throughput and a lower transmit power of the D2D system.

The paper by P. Sroka and A. Kliks, entitled "Playing Radio Resource Management Games in Dense Wireless $5 G$ Networks" proposes an efficient and flexible tool for interference mitigation in ultradense heterogeneous cellular $5 \mathrm{G}$ networks. Authors study, via simulation, various gametheory based algorithms which concentrate on the optimization of the overall base station energy consumption. Simulation results verify that the adopted game-theoretic algorithms are very promising solutions for interference mitigation outperforming the algorithm proposed for LTEAdvanced in terms of the achieved spectral efficiency.

The paper by S. Hanczewski et al. entitled "Convolution Model of a Queueing System with the cFIFO Service Discipline" proposes an approximate convolution model of a multiservice queueing system that services a mixture of independent multiservice Bernoulli-Poisson-Pascal call streams under the continuous First-In-First-Out (cFIFO) service discipline. The accuracy of the proposed convolution model is verified by simulation experiments for a number of queueing systems.

The paper by F. Alsewaidi et al. entitled "Enhancing Radio Access Network Performance over LTE-A for Machine-toMachine Communications under Massive Access" proposes three methods to enhance RAN performance for Machineto-Machine (M2M) communications over the LTE-A standard. The first method employs a different value for the physical Random Access Channel (RACH) configuration index to increase random access opportunities. The second method addresses a heterogeneous network by using a number of picocells to increase resources and offload control traffic from the macro base station. The third method involves aggregation points and addresses their effect on RAN performance. The results presented in the paper confirmed that the proposed methods improved RACH performance in terms of access success probability and average delay.

The paper by J. Säe and J. Lempiäinen, entitled "Maintaining Mobile Network Coverage Availability in Disturbance Scenarios," studies the case of disturbance and disaster scenarios in mobile networks. Authors perform various simulation scenarios under different network layouts with the aim of maintaining the availability of cellular networks in disturbance scenarios. Simulation results show how the mobile network availability duration can be sustained by selecting a set of evolved node $B(e N B)$ sites to operate at a time and still maintain a reasonable service level and availability in disturbance scenarios.

In the paper "Macrocell Protection Interference Alignment in Two-Tier Downlink Heterogeneous Networks" by J. Seo et al., the MCP-IA method to limit interference problems in two-tier (picocells and macrocells) MIMO networks was elaborated. Due to the proposed solution, the additional array and diversity gains, in comparison to the results obtained on the basis of existing interference alignment solutions, can be obtained for the macro users. The performance of the proposed method was evaluated in a simulation environment, at the link level as well as at the system.

The paper "LDPC Decoding on GPU for Mobile Device" by Y. Lu et al. proposed a software multicode word parallel LDPC decoder, exploiting OpenCL based graphics processing units built-in into mobile devices. Software realization of LDPC decoding ensures a possibility of dynamic change of code length, code rate, and the number of iterations, in order to tune to networks' conditions. The tests led in the experimental test-bed confirmed high performance of the proposed solution and confirms a possibility of its application for large file transmission and delay-sensitive services like video calling in mobile networks.

The paper "Latent Clustering Models for Outlier Identification in Telecom Data" by Y. Ouyang et al. deals with the problem of fast and robust identification of unexpected traffic streams in high-speed mobile networks, generated in the case of malicious attacks or technical problems. As the solution to this problem, the authors selected an approach based on clustering models and proposed the application of Gaussian Probabilistic Latent Semantic Analysis (GPLSA) and timedependent Gaussian Mixture Models (timeGMM). The comparison of the efficiency of the methods was performed in a simulation environment.

Mariusz Głąbowski Haris Gacanin Ioannis Moscholios Piotr Zwierzykowski 

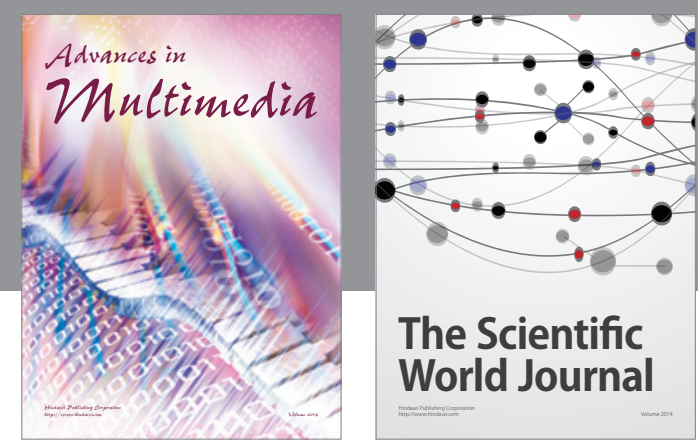

The Scientific World Journal
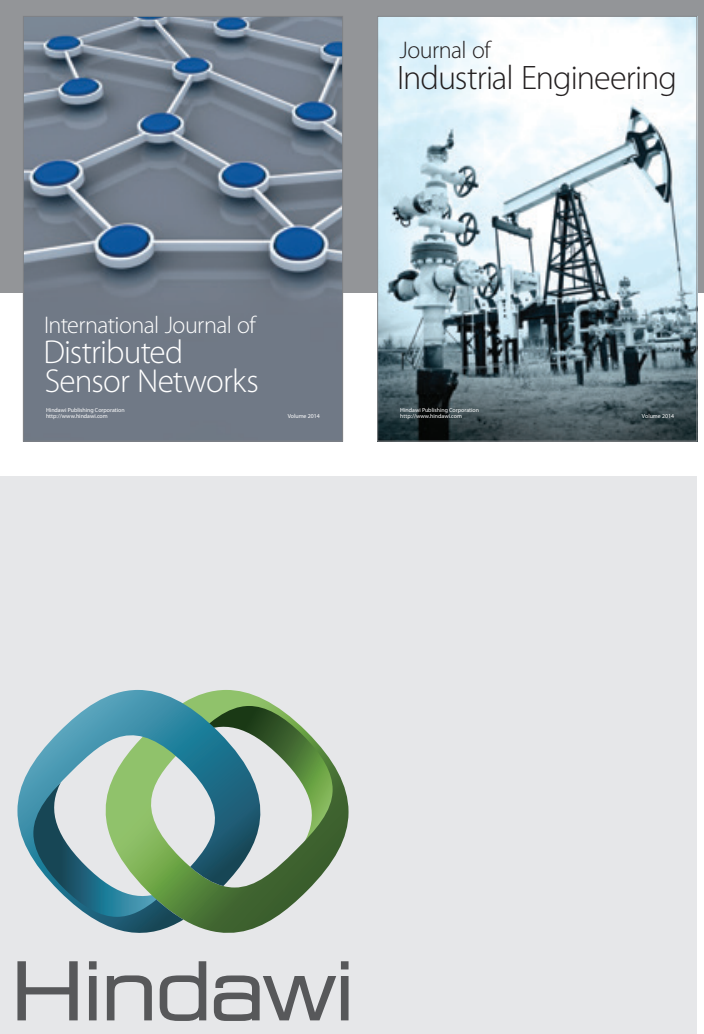

Submit your manuscripts at

https://www.hindawi.com

\section{Computer Networks} and Communications
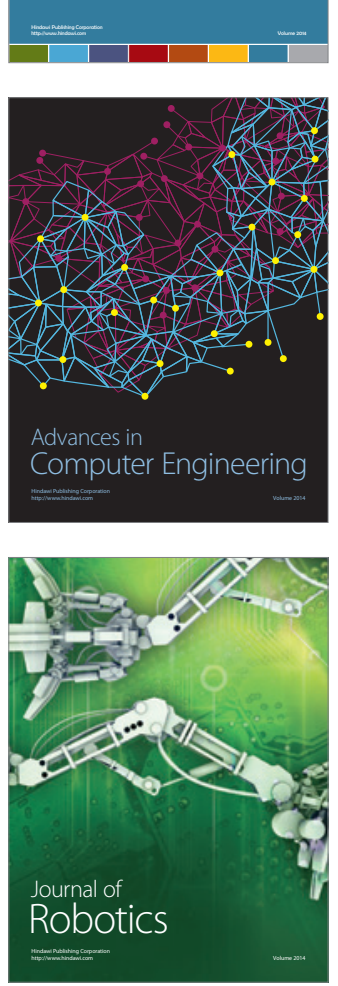
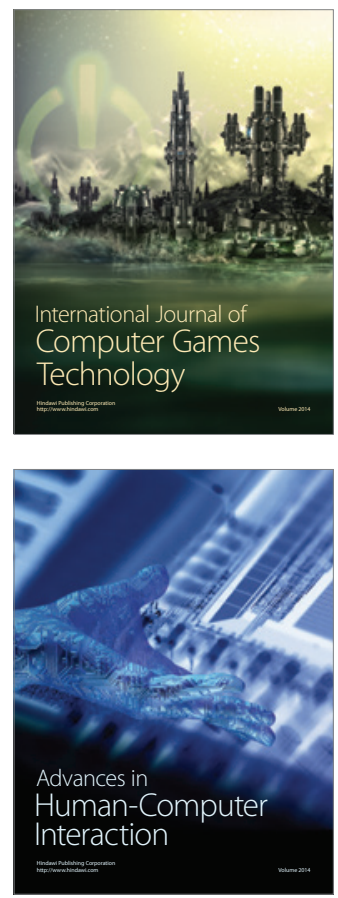
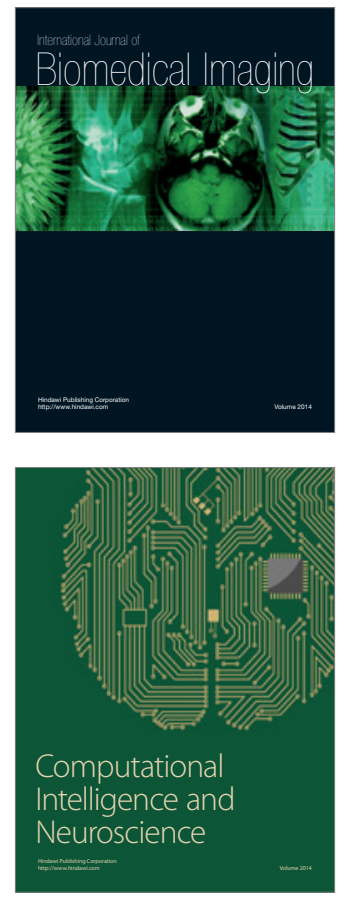
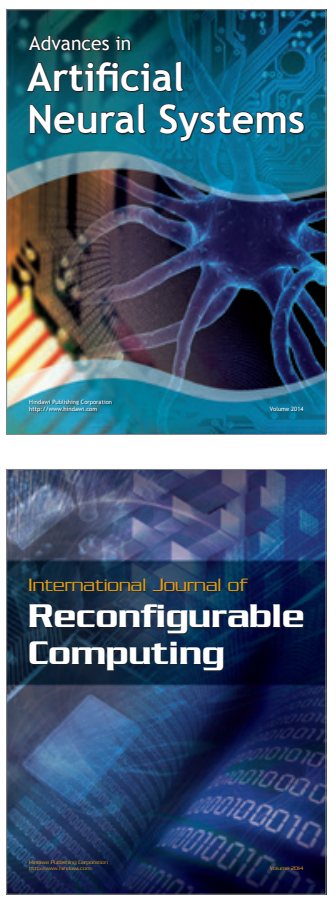
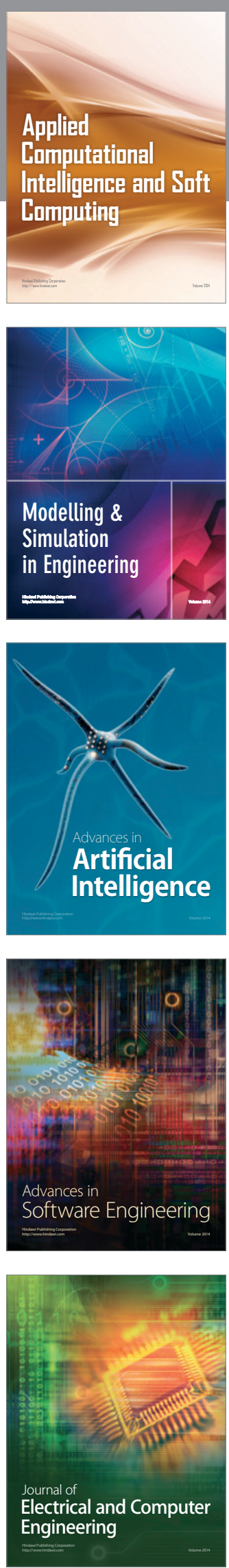